Rubrikherausgeber:

Tom Schaberg, Rotenburg (Koordination)

Roland Buhl, Mainz

Adrian Gillissen, Leipzig
Karl Häußinger, Gauting

Rolf Merget, Bochum

Claus Vogelmeier, Marburg

T. O. F. Wagner, Frankfurt
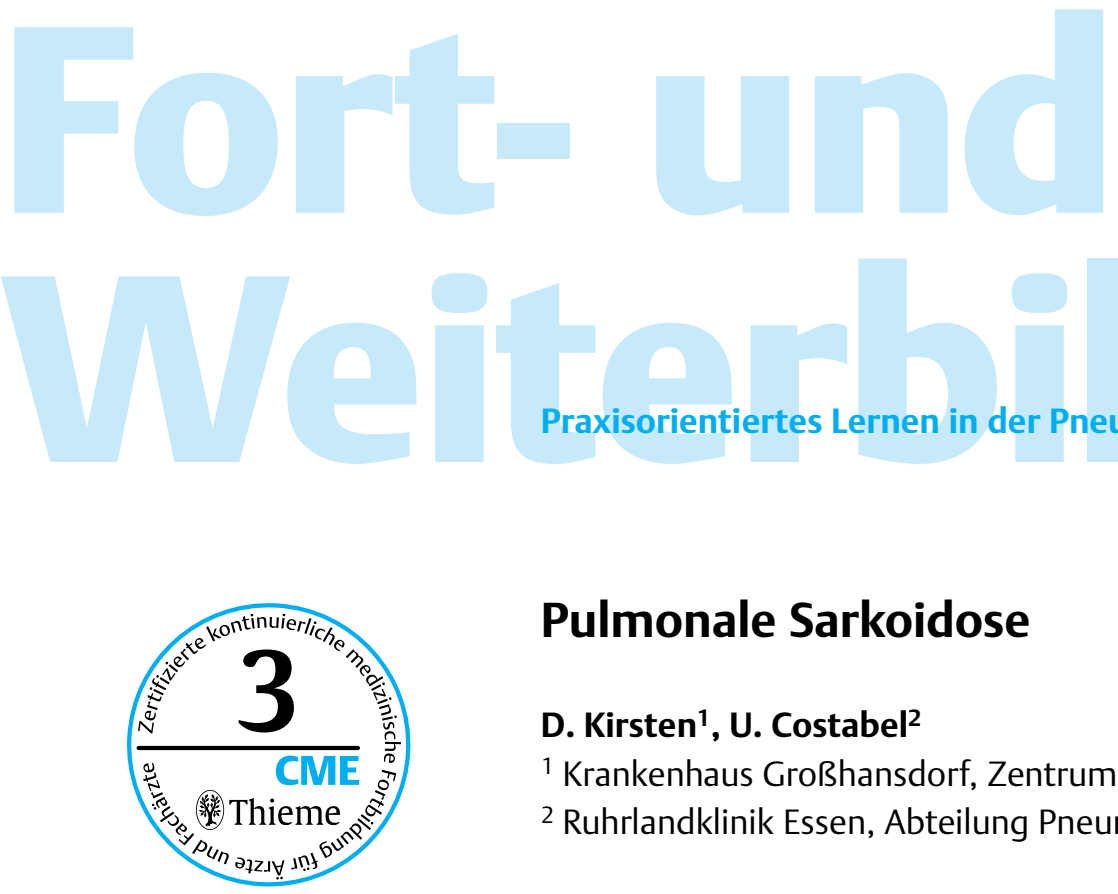

\title{
Pulmonale Sarkoidose
}

\section{Kirsten ${ }^{1}$, U. Costabel ${ }^{2}$}

${ }^{1}$ Krankenhaus Großhansdorf, Zentrum für Pneumologie und Thoraxchirurgie

2 Ruhrlandklinik Essen, Abteilung Pneumologie/Allergologie

\section{Weiterbildungsziele}

In diesem Beitrag sollen folgende Weiterbildungsziele vermittelt werden:

\section{Epidemiologie}

2. Ätiologie und Pathogenese

2.1 Ätiologie

2.2 Pathogenese

3. Histopathologie

4. Symptomatologie und Klinik

4.1 Akute Sarkoidose

4.2 Chronische Sarkoidose

5. Diagnostische Maßnahmen bei Sarkoidose

5.1 Obligate Maßnahmen

5.2 Der Patient ohne Histologie

5.3 Weiterführende Diagnostik

\section{Aktivitätsdiagnostik}

7. Verlauf und Prognose

7.1 Ungünstige prognostische Faktoren

7.2 Verlaufsbeobachtung

8. Therapie

8.1 Therapie der Lungensarkoidose

8.2 Extrathorakale Manifestation

8.3 Alternativen zur Kortikosteroidtherapie

9. Schlußbemerkung 
Die Sarkoidose ist eine granulomatöse Systemerkrankung mit bislang unbekannter Ätiologie.

Häufigkeitsgipfel zwischen dem 20. und 40. Lebensjahr.

Gesichert ist eine genetische Prädisposition.

Wahrscheinlich ein polyätiologisches Syndrom, welches durch unbekannte inhalierbare Antigene ausgelöst wird.

Im Zentrum des immunologischen Geschehens bei Sarkoidose stehen die Alveolarmakrophagen und die T-Helfer-Lymphozyten vom Th1-Typ.
Die Sarkoidose ist eine granulomatöse Systemerkrankung mit bislang unbekannter Ätiologie. Fast immer sind die hilären und mediastinalen Lymphknoten befallen, häufig finden sich auch extrapulmonale Manifestationen. Die Diagnose gilt aus gesichert, wenn typische klinisch-radiologische Befunde ihre Bestätigung durch den Nachweis nicht-verkäsender epitheloidzelliger Granulome finden. Für die Sarkoidose charakteristisch sind immunologische Befunde wie die Unterdrückung der Tuberkulin- und anderer Hautreaktionen vom verzögerten Typ verbunden mit einer verstärkten Immunreaktion von Th1-Typ in den befallenen Organen.

Die erste Erwähnung einer Sarkoidose (einer Hautsarkoidose) geht auf J. Hutchinson auf das Jahr 1877 zurück. 1899 beschrieb Cäsar Boeck Hautveränderungen, die er als multiples benignes Sarkoid bezeichnete. Sein Name diente viele Jahrzehnte als Krankheitssynonym.

\section{Epidemiologie}

Die Sarkoidose tritt weltweit auf. Die Prävalenz weist ein gewisses Nord-Süd-Gefälle auf (Schweden 64/100 000; Spanien 4/100000). In Deutschland rechnet man mit einer Inzidenz von ca. 10 Erkrankten auf 100000 Einwohner [1]. Die Dunkelziffer ist jedoch groß, da bei asymptomatischem Verlauf die Krankheit lediglich durch ThoraxröntgenUntersuchungen aufgedeckt werden kann. In Ländern mit geringem Röntgen-Screening sind demnach die Erkrankungsfälle deutlich seltener.

Die Sarkoidose tritt in jedem Lebensalter auf, wobei ein Häufigkeitsgipfel zwischen dem 20. und 40. Lebensjahr besteht. Erkrankungen im Kindesalter und oberhalb des 65. Lebensjahres sind selten. In Deutschland gibt es keinen wesentlichen Unterschied in der Häufigkeit der Erkrankung zwischen Männern und Frauen [1,2].

Gesichert ist eine genetische Prädisposition. Dafür sprechen Unterschiede in der Häufigkeit und Ausprägung der Sarkoidose bei verschiedenen Bevölkerungsgruppen. So erkranken Afro-Amerikaner häufiger an einer prognostisch ungünstigen chronisch-progredienten Form, während in den skandinavischen Ländern die akute Form (LöfgrenSyndrom) häufiger vorkommt. Die Sarkoidose kann auch familiär gehäuft auftreten [3].

\section{2 Ätiologie und Pathogenese}

\section{1 Ätiologie}

Die Ätiologie der Sarkoidose ist bislang unbekannt. Unterschiedliche Krankheitsverläufe lassen Spekulationen zu, nach denen es sich wahrscheinlich um ein polyätiologisches Syndrom handelt, welches durch unbekannte inhalierbare Antigene ausgelöst wird. Für eine genetisch bedingte Disposition sprechen u. a. unterschiedliche HLA-Konstellationen bei akuter und ausgedehnter systemischer Erkrankung.

Die Tab. 1 zeigt die ätiologischen Faktoren, die bei der Auslösung von Sarkoidose bislang ins Spiel gebracht wurden (nach ATS/ERS/WASOG-Statement 1999).

\subsection{Pathogenese}

Im Zentrum des immunologischen Geschehens bei Sarkoidose stehen die Alveolarmakrophagen und die T-Helfer-Lymphozyten vom Th1-Typ. Die von ihnen sezernierten Zytokine und anderen Mediatoren bedingen einerseits die Granulombildung und andererseits sind sie an der Fibroblastenstimulation und damit der Fibrosierung beteiligt. 
TNF- $\alpha$ kommt offenbar die bedeutendste Rolle bei der Granulombildung zu.

Differenzialdiagnostisch ist die fehlende Verkäsung besonders in Bezug auf die Differenzialdiagnose zur Tuberkulose wichtig.

Die Granulome in der Lunge folgen meistens dem bronchovaskulären Bündel, so dass eine so genannte lymphangitische Verteilung resultiert.
Tab. 1 Mögliche ätiologische Faktoren bei Sarkoidose

\begin{tabular}{lll}
\hline infektiöse Ursachen & anorganische Ursachen & organische Ursachen \\
\hline & & \\
\hline „Viren“ & Aluminium & Pinienpollen \\
$\begin{array}{l}\text { Propionibacterium acnes } \\
\text { Mycobacterium tuberculosis, }\end{array}$ & Talk & Erdbestandteile (Lehm) \\
$\begin{array}{l}\text { andere Mycobacterien } \\
\text { Mycoplasmen }\end{array}$ & Zirkonium & \\
$\begin{array}{l}\text { Chlamydien } \\
\text { Rickettsien }\end{array}$ & & \\
zellwandlose Bakterien & & \\
\hline
\end{tabular}

Die aktivierten T-Zellen sezernieren Interferon- $\gamma$, Interleukin-2 und chemotaktische Faktoren für Blutmonozyten. Während in der Lunge die Lymphozyten akkumulieren, kommt es im peripheren Blut zu einer T-Zell-Lymphopenie, die letztendlich für die negativen Teste vom verzögerten Typ (z.B. Tuberkulintest) verantwortlich ist. Die aktivierten Makrophagen produzieren zahlreiche proinflammatorische Zytokine. Dabei kommt TNF- $\alpha$ offenbar die bedeutendste Rolle bei der Granulombildung zu.

\section{Histopathologie}

Das histopathologische Substrat der Sarkoidose ist das Epitheloidzellgranulom. Charakteristisch sind kompakte, gleichförmig aussehende nicht-verkäsende Granulome, die aus Epitheloidzellen bestehen und von einem Lymphozytenwall umgeben sind. Differenzialdiagnostisch ist die fehlende Verkäsung besonders in Bezug auf die Differenzialdiagnose zur Tuberkulose wichtig. Die darüber hinaus vorkommenden mehrkernigen Riesenzellen vom Langerhans-Typ können Einschlusskörperchen, wie Schaumannkörperchen oder Astereoidkörperchen aufweisen. Die Granulome können sich im Verlauf der Erkrankung zurückbilden unter Hinterlassung zarter Narbenbildung. Sie können aber auch über Jahre und Jahrzehnte persistieren ohne sich zu verändern, im prognostischen ungünstigen Fall jedoch auch zunehmen oder zu deutlicher Fibrosierung führen, die zur Zerstörung des umliegenden Gewebes führt. Die Granulome in der Lunge folgen meistens dem bronchovaskulären Bündel, so dass eine so genannte lymphangitische Verteilung resultiert.

Die Abb.1 zeigt ein typisches nicht-verkäsendes Epitheloidzellgranulom.

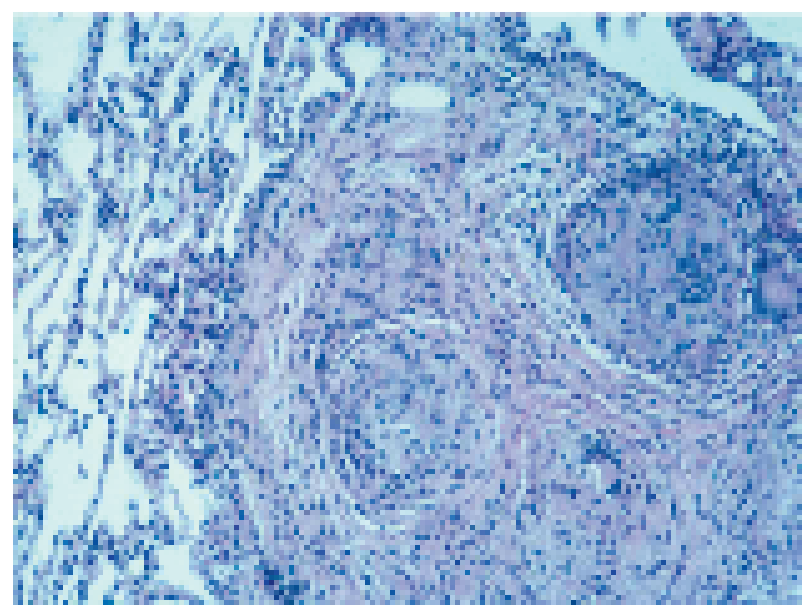

Abb. 1 Typisches nicht verkäsendes Epitheloidzellgranulom. 
Die Sarkoidose manifestiert sich in zwei Verlaufsformen, die sich in Bezug auf Prognose und Therapiebedürftigkeit grundsätzlich unterscheiden. Bezüglich der Symptomatologie wird davon ausgegangen, dass die Hälfte aller Krankheitsfälle asymptomatisch sind.

\subsection{Akute Sarkoidose}

Die akute Sarkoidose (in Deutschland 10 bis $20 \%$ aller Erkrankungsfälle) wird in klassischer Ausprägung auch als Löfgren-Syndrom bezeichnet, da Sven Löfgren als erster diese Krankheitsform als eigene Entität von der Tuberkulose abgrenzte.

Das Löfgren-Syndrom ist ein schweres Krankheitsgeschehen mit grippeartigen Symptomen, Abgeschlagenheit, Appetitlosigkeit und teilweise hohem Fieber. Es findet sich die klassische Trias: „Bihiläre Adenopathie“, ausgeprägte Arthritis meist der oberen Sprunggelenke, Erythema nodosum meistens an den Streckseiten beider Unterschenkel (Abb. 2).

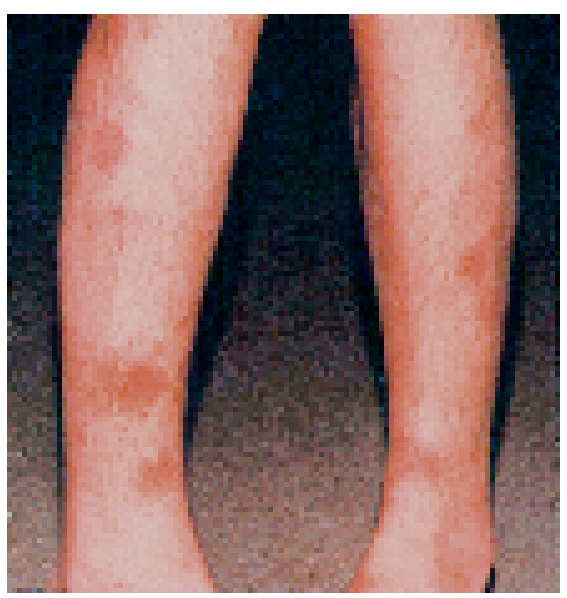

Abb. 2 Erythema nodosum.

Therapeutisch werden bei der akuten Sarkoidose nicht-steroidale Antiphlogistika eingesetzt.

Bei oberer Sprunggelenksarthritis und Erythema nodosum immer auch an Sarkoidose denken!

Ausgeprägte Röntgenveränderungen stehen oft im Missverhältnis zur Beschwerdearmut der Patienten!
Die Sarkoidose sollte in Mitteleuropa immer in die Differenzialdiagnose unklarer Fieberzustände mit Erythema nodosum einbezogen werden. Ein rheumatisches Fieber ist abzugrenzen. Die akute Form betrifft vorwiegend junge Frauen. Es gibt einen jahreszeitlichen Häufigkeitsgipfel in den Monaten März und April.

Die Prognose ist in aller Regel gut, da bis zu 90\% der Patienten innerhalb von zwei Jahren eine Spontanheilung erleben. Therapeutisch werden bei der akuten Sarkoidose nicht-steroidale Antiphlogistika eingesetzt, Cortisonpräparate sind nur ausnahmsweise bei stärksten Beschwerden notwendig, wenn die symptomatische Behandlung mit nicht-steroidalen Antiphlogistika nicht ausreichend wirkt. Extrem selten sind Rezidive eines Erythema nodosum oder eines Löfgren-Syndroms.

\subsection{Chronische Sarkoidose}

Die chronische Sarkoidose verläuft im Gegensatz zur akuten Sarkoidose oft über lange Zeit relativ symptomarm, bei bis zu 50\% völlig asymptomatisch. Die Tab. 2 zeigt die allgemeinen Symptome bei Sarkoidose in Anlehnung an Drent und Wirnsberger [4]. Aber auch bei den Patienten, die nahezu asymptomatisch sind, können ausgeprägte Röntgenveränderungen diagnostiziert werden (Abb.3). 
Tab. 2 Chronische Sarkoidose nach Drent u. Mitarb. [4]

allgemeine Symptome:

$71 \%$ Müdigkeit

$70 \%$ Atembeschwerden

$52 \%$ Gelenkbeschwerden

$39 \%$ Muskelschmerzen

$27 \%$ Thoraxschmerzen

$15 \%$ allgemeine Schwäche, Nachtschweiß, Gewichtsverlust, selten Fieber

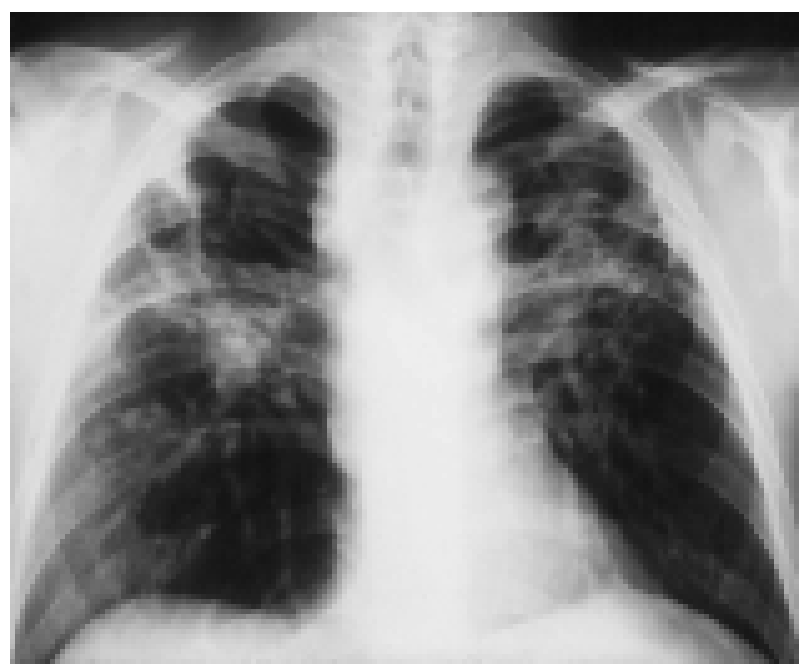

Abb. 3 Röntgen-Thorax Typ III bei Sarkoidose.

Tab. 3 Organmanifestationen nach Deutschen Empfehlungen zur Diagnostik und Therapie der Sarkoidose 1998 [5]

\begin{tabular}{|l|l|}
\hline mediastinale Hiluslymphknotenvergrößerungen & $100 \%$ \\
\hline Arthritis & $60-80 \%$ \\
\hline Erythema nodosum & $60-80 \%$ \\
\hline bei chronischer Sarkoidose & \\
\hline mediastinale Lymphknoten & $100 \%$ \\
\hline Lunge & $90 \%$ \\
\hline Milz & $50-70 \%$ \\
\hline Leber & $50-70 \%$ \\
\hline Augen & $20-50 \%$ \\
\hline Bronchialschleimhaut & $50 \%$ \\
\hline periphere Lymphknoten & $30 \%$ \\
\hline Parotis (Speicheldrüsen) & $30 \%$ \\
\hline Haut & $20 \%$ \\
\hline Herz & $20 \%$ \\
\hline Muskulatur & $20 \%$ \\
\hline Nervensystem & $15 \%$ \\
\hline Niere & $10 \%$ \\
\hline Knochen & $10 \%$ \\
\hline
\end{tabular}

Die Prozentangaben sind als derzeit maximal beschriebene Häufigkeiten anzusehen.

Die Häufigkeit der verschiedenen Organmanifestationen ist in der Tab. 3 dargestellt. Diese Angaben haben alle einen orientierenden Charakter, da sie abhängig sind von der Intensität der Diagnostik und auch von speziellem diagnostischem Interesse der Untersucher. Prinzipiell kann jedes Organ befallen sein [5]. 
Radiologisch wird die Sarkoidose in 4 Typen eingeteilt.

\section{Diagnostische Maßnahmen bei Sarkoidose}

Der Diagnosebeweis kann nicht auf ein einziges diagnostisches Verfahren reduziert werden [3].

Die Diagnose der Sarkoidose stützt sich auf:

- passende klinische und/oder radiologische Befunde

- den histologischen Nachweis von nicht verkäsenden Granulomen sowie

- den Ausschluss anderer Granulomatosen

\subsection{Obligate Maßnahmen}

\section{Klinische Untersuchung}

Hier muss vor allem auf pulmonale Symptome geachtet sowie gezielt nach extrathorakalen Befunden gesucht werden, wobei besonders Augensymptome, Hautveränderungen und Herzrhythmusstörungen zu beachten sind.

\section{Röntgenuntersuchung der Thoraxorgane in 2 Ebenen}

Radiologisch wird die Sarkoidose in 4 Typen eingeteilt [6] (siehe Tab. 4). Eine charakteristische radiologische Entwicklung im Krankheitsverlauf kann die Diagnose ohne histologische Bestätigung zulassen [7]. Als besonderer Erscheinungstyp ist die Rundherdsarkoidose zu erwähnen, welche zunächst an Lungenmetastasen eines unbekannten Primärtumors denken lässt.

Tab. 4 Röntgentypen der Sarkoidose: Häufigkeit, spontane Regredienz, nebst Differenzialdiagnosen

\begin{tabular}{|c|c|c|c|c|}
\hline Typ & Röntgenaspekt & $\begin{array}{l}\text { Häufigkeit } \\
\text { (\%) }\end{array}$ & $\begin{array}{l}\text { Remission } \\
\text { (\%) }\end{array}$ & Differenzialdiagnosen \\
\hline I & bihiläre Adenopathie & 50 & bis 70 & $\begin{array}{l}\text { Tuberkulose, Silikose, Bruzellose, } \\
\text { Bronchialkarzinom, malignes Lym- } \\
\text { phom }\end{array}$ \\
\hline II & $\begin{array}{l}\text { bihiläre Adenopathie } \\
\text { und Lungeninfiltrate }\end{array}$ & 25 & bis 50 & $\begin{array}{l}\text { wie oben und Lymphangiosis carci- } \\
\text { nomatosa }\end{array}$ \\
\hline III & $\begin{array}{l}\text { Lungenparenchym- } \\
\text { infiltrate ohne Hilus- } \\
\text { lymphknotenvergröße- } \\
\text { rung }\end{array}$ & 15 & bis 20 & $\begin{array}{l}\text { Berylliose, Tuberkulose, Silikose, Al- } \\
\text { veolitis, Lungenfibrose, Histiozytosis } \\
\text { X, Kollagenosen, Lymphangiosis car- } \\
\text { cinomatosa }\end{array}$ \\
\hline IV & Fibrosestadium & 10 & bis 0 & $\begin{array}{l}\text { Berylliose, Tuberkulose, Alveolitis, } \\
\text { bullöses Emphysem, Bronchiektasen }\end{array}$ \\
\hline
\end{tabular}

\section{Lungenfunktionsdiagnostik}

Hier kommen Spirometrie, Bestimmung des arteriellen $\mathrm{O}_{2}$-Partialdruckes in Ruhe und unter Belastung und/oder Diffusionskapazitätsbestimmung zur Anwendung.

Diese Untersuchungen sind bei allen Patienten anfangs indiziert, um die Lungenfunktion zum Zeitpunkt der Diagnosestellung festzuhalten und an den Ausgangswerten die Verschlechterung oder Verbesserung im Krankheitsverlauf zu messen. Bei Patienten mit Typ-I-Sarkoidose finden sich nur bei 20\% Lungenfunktionsstörungen, bei den sonstigen Röntgentypen bei 40-70\%. Die empfindlichsten Parameter sind Diffusionskapazität und Vitalkapazität. Bei bis zu 30\% der Fälle werden auch obstruktive Ventilationsstörungen festgestellt, bei $25 \%$ eine bronchiale Hyperreagibilität $[8,9]$. 
Unter Kortisontherapie normalisieren sich ACE-Werte, ein Rezidiv kann mit einem Anstieg des ACE verbunden sein.

Jeder Sarkoidosepatient muss zum Augenarzt.

Die Methode der Wahl stellt heute die Bronchoskopie mit Schleimhautbiopsie, transbronchialer Lungenbiopsie und bronchoalveolärer Lavage (BAL) dar.

Cave: Leberbiopsien sind nicht zu empfehlen, denn hier sind andere granulomatöse Erkrankungen zu häufig und führen zu differenzialdiagnostischen Problemen.

Bei Patienten mit klassischem Löfgren-Syndrom kann in der Regel auf eine Biopsie verzichtet werden.

\section{Elektrokardiogramm}

Bei Herzrhythmusstörungen und Blockbildern sind zusätzlich Langzeit-EKG, Echokardiographie und weitere Maßnahmen angebracht [10].

\section{Tuberkulintest}

Dieser ist bei Sarkoidose meist negativ.

\section{Labor}

Das Serum-ACE wird von Makrophagen und Granulomzellen gebildet. Die Sensitivität beträgt etwa 60 -70\%, die Spezifität $80 \%$. Das ACE eignet sich für Verlaufsuntersuchungen, ein Abfall des Enzyms bei unbehandelter Sarkoidose ist mit einer Remission verbunden. Unter Kortisontherapie normalisieren sich ACE-Werte, ein Rezidiv kann mit einem Anstieg des ACE verbunden sein.

Ferner werden Blutbild mit Differenzierung, Leberenzyme, Retentionswerte, Kalzium im Serum, ggf. im 24-h-Urin, bestimmt.

Eine Hyperkalzämie kommt bei etwa 10\% der Sarkoidosen vor, resultiert aus einer gesteigerten intestinalen Absorption von Kalzium aufgrund einer Überproduktion des 1,25-Dihydroxy-Vitamin- $\mathrm{D}_{3}$ (Calcitriol), der aktiven Form des Vitamin $\mathrm{D}_{3}$, durch aktivierte Makrophagen und stellt eine absolute Behandlungsindikation dar.

\section{Augenärztliche Untersuchung}

Jeder Sarkoidosepatient muss initial ophthalmologisch untersucht werden, da die Augenbeteiligung asymptomatisch verlaufen kann.

\section{Bioptische Maßnahmen}

Eine Organbiopsie kann aus einfach zugänglichen Bereichen erfolgen, z. B. aus der Haut (nicht aus Erythema nodosum!), frischen Granulomen in Narben oder aus peripheren Lymphknoten.

Die Methode der Wahl stellt heute die Bronchoskopie mit Schleimhautbiopsie, transbronchialer Lungenbiopsie und bronchoalveolärer Lavage (BAL) dar. Die Trefferquote der transbronchialen Biopsie erreicht bis zu $80 \%$ bei Entnahme von 4-5 Biopsien. Die Bronchialschleimhautbiopsie weist auch bei makroskopisch unauffälliger Bronchialschleimhaut bei bis zu 40-60\% histologisch Granulome auf. Die Trefferquote der perbronchialen Lymphknotenbiopsie beträgt bis $\mathrm{zu} 50 \%$ [11]. In der BAL ist ein CD4/CD8-Quotient von über 3,5 hochspezifisch für die Sarkoidose, so dass er mit dem Nachweis von Epitheloidzellgranulomen gleichgesetzt werden kann. Die Sensitivität beträgt jedoch nur 50-60\% [12].

Nur wenn diese Methoden diagnostisch nicht hilfreich sind, sollte noch eine Mediastinoskopie bzw. eine thorakoskopische oder chirurgische Lungenbiopsie durchgeführt werden. So genannte Suchbiopsien sollten nicht erfolgen.

\subsection{Der Patient ohne Histologie}

Einige Patienten lehnen die Biopsie ab, bei anderen kann die Lungenbiopsie mit erhöhtem Risiko verbunden sein. Klinische und radiologische Befunde allein sind bei Patienten im Typ I von hoher diagnostischer Zuverlässigkeit (98\%), auch im Typ II noch ausreichend (89\%), jedoch deutlich weniger akkurat im Typ III (52\%) oder gar im Typ 0 (23\%) [3]. 
CT-Thorax soll nur gezielt eingesetzt werden.

\subsection{Weiterführende Diagnostik Computertomographie des Thorax}

Diese Untersuchung sollte nur gezielt eingesetzt werden.

Indikationen für ein Thorax-CT sind:

- atypische klinische und/oder Thoraxröntgenbefunde,

- Verdacht auf Komplikationen, wie Bronchiektasen, Aspergillom, Taktionsemphysem oder Malignomverdacht,

- normales Röntgenbild bei klinisch starkem Verdacht auf das Vorliegen einer Sarkoidose.

Charakteristische Zeichen im Thorax-CT sind [13]:

- multiple kleine Knötchen mit ausgesprochener bronchovaskulärer und subpleuraler Verteilung,

- verbreiterte interlobuläre Septen,

- Zerstörung der Lungenarchitektur,

- größere Verschattungen durch Konfluenz einzelner Granulome.

Die Abb. 4 zeigt ein typisches CT-Bild einer Sarkoidose.

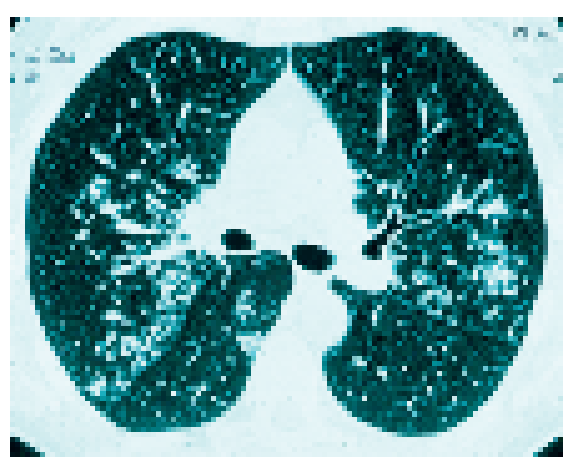

Abb. 4 Typisches CT-Bild einer Sarkoidose.

\section{Fakultative Untersuchungen}

Diese sind erforderlich bei Verdacht auf bestimmte Organsarkoidosen wie:

- Herzbefall: Langzeit-EKG, Echokardiographie, Magnetresonanztomographie. Myokardbiopsie nur ausnahmsweise bei differenzialdiagnostischen Problemen zur Myokarditis oder zu Speicherkrankheiten.

- Neurosarkoidose: Magnetresonanztomographie, ACE im Liquor.

- Augenbeteiligung: evtl. zusätzlich Fluoreszenzangiographie.

- Sicca-Syndrom und Verdacht auf Tränen- und Speicheldrüsensarkoidose: Biopsie, ggf. Galliumszintigraphie.

- Nasennebenhöhlenbefall: Biopsie, ggf. Galliumszintigraphie

Neben der Lungensarkoidose sind Augen- und Hautsarkoidose die häufigsten extrapulmonalen Manifestationen. Beide Manifestationen sind klinisch gut zu diagnostizieren und im Verlauf zu beobachten.

Schwieriger ist die Situation bei der Sarkoidosemanifestation an Herz, Hirn, Knochen und Muskulatur. Hier hat sich in letzter Zeit besonders die Magnetresonanztomographie (MRT) als Diagnostikum der Wahl etabliert [10]. Voraussetzung ist selbstverständlich ein klarer klinischer Verdacht. Der besteht bei der Herzsarkoidose in erster Linie in Herzrhythmusstörungen, die anderweitig nicht geklärt werden können, zumal bei jungen Erwachsenen. Hier sind prinzipiell alle EKG-Störungen möglich. Bei der Neurosarkoidose sind zentrale Prozesse oder periphere Hirnnervenmanifestationen, wie Fazialisbeteili- 
Die Beurteilung der Krankheitsaktivität erfolgt nach wie vor aufgrund des klinischen Bildes (Symptome), der Verlaufstendenz des Röntgenbildes (Progredienz) und der Lungenfunktion (Verschlechterung).

Die Sarkoidose zeichnet sich durch eine hohe spontane Remissionsrate aus.

Die Prognose quoad vitam ist gut, die sarkoidosebezogene Mortalität niedrig. Sie beträgt 1 bis $5 \%$. gung zu nennen. Ein klinisch erkennbarer Befall des zentralen Nervensystems findet sich in weniger als $10 \%$. Auch hier ist das MRT die diagnostische Methode der Wahl. Auffällige klinische Befunde sind hier Kopfschmerz, Herdsymptome, sowie Hirnnervenausfälle.

\section{Aktivitätsdiagnostik}

Der Begriff „Aktivität“ wird bei Sarkoidose häufig gebraucht, sollte allerdings nicht mit dem Ausmaß der Erkrankung (d.h. der Zahl der befallenen Organe, der Granulomdichte innerhalb eines betroffenen Organes) verwechselt werden, sollte auch nicht mit ungünstiger Prognose verbunden werden (z.B. hat die hoch aktive akute Sarkoidose, das Löfgren-Syndrom, die beste Prognose), und schließlich auch nicht mit der Notwendigkeit, eine Kortikosteroidtherapie zu beginnen, da auch aktive Sarkoidosen in hohem Prozentsatz spontan ausheilen können [14].

Für die klinische Routine sind derzeit die körperliche Untersuchung sowie die Symptomatologie mit Frage nach den o.g. Symptomen mit entscheidend und insbesondere deren Schweregrad im Verlauf. Daneben sind weitere Aktivitätszeichen die radiologische Befundentwicklung und der Verlauf der Lungenfunktion. An serologischen Markern hat sich in der Routine das Serum ACE behauptet, das zur Beobachtung des Therapieerfolges eingesetzt wird.

Im klinischen Alltag kann möglicherweise in Zukunft der so genannte ACE-Deletion-Insertions-Polymorphismus Beachtung finden. Bei homozygotem II-Typ können auch bei großer Krankheits-Aktivität niedrige ACE-Werte möglich sein, was dann klinisch zu beachten ist [15]. In neuerer Zeit hat sich unter den einfach zu erhebenden Aktivitätsparametern besonders die Bestimmung des IL-2-Rezeptors durchgesetzt [16].

\section{Verlauf und Prognose}

Grundsätzlich gilt, dass die Prognose umso günstiger ist, je akuter die Erkrankung beginnt. Dies gilt in erster Linie für Erkrankungsverläufe mit akutem Beginn oder dem so genannten Löfgren-Syndrom. Hier ist, wie bereits oben ausgeführt, die Prognose besonders günstig, da innerhalb von zwei Jahren alle Krankheitssymptome verschwinden. In seltenen Fällen persistiert die bihiläre Adenopathie noch etwas länger. Bei der chronischen Sarkoidose finden sich abhängig vom Röntgentyp unterschiedliche Spontanremissionsraten, die in Tab. 3 niedergelegt sind.

Die Prognose ist bei schweren extrathorakalen Organmanifestationen, wie Herzbeteiligung, Leberbeteiligung, Milzbeteiligung, Nervenbeteiligung, generell schlechter als bei symptomarmen reinen Lungensarkoidosen des Röntgentypes II und III. Die Prognose quoad vitam ist gut, die sarkoidosebezogene Mortalität niedrig. Sie beträgt 1 bis $5 \%$ $[17,18]$. Die Todesfälle bei Sarkoidose sind durch die Herzbeteiligung oder durch die chronisch-progrediente respiratorische Insuffizienz bei Patienten mit Röntgentyp IV bedingt.

\subsection{Ungünstige prognostische Faktoren}

Ungünstige prognostische Faktoren, die mit chronischem oder progredientem Verlauf assoziiert sind, umfassen [3]:

- Lupus pernio

- chronische Uveitis

- höheres Lebensalter (> 40 Jahre)

- chronische Hyperkalziämie 
Ziel der Therapie ist, Symptome zu lindern und den Funktionsverlust zu antagonisieren.

Therapie der Wahl: Gabe von oralem Prednison, initial 20-40 mg.

Die Therapiedauer beträgt zwischen 6 und 12 Monaten.
- Nephrokalzinose

- schwarze Rasse

- progrediente Lungensarkoidose

- Nasenschleimhautbeteiligung

- zystische Knochenläsionen

- Neurosarkoidose

- Herzbefall

- chronische respiratorische Insuffizienz

\subsection{Verlaufsbeobachtung}

Wichtig ist zur Kennzeichnung des individuellen Verlaufes die Beobachtung über mindestens drei Jahre nach erfolgreichem Therapieende. Dies heißt, es darf kein Rezidiv nach Absetzen der Therapie erfolgen. Hat sich eine derartige Stabilität des Krankheitsverlaufes - auch mit Restbefunden im Thorax-Röntgenbild - eingestellt, sind weitere Routine-Beobachtungen nicht nötig, sonder nur noch symptomgebunden zu fordern [3]. Patienten mit persistierenden chronischen Symptomen müssen meist lebenslang betreut werden. Dies trifft selbstverständlich auch für Patienten mit ausgeprägten extrathorakalen Manifestationen der Sarkoidose zu.

\section{Therapie}

\subsection{Therapie der Lungensarkoidose}

Die Therapie der Lungensarkoidose ist nicht von den Röntgenbefunden, sondern einzig und allein von den Symptomen und der Funktionseinschränkung abzuleiten [5]. Ziel der Therapie ist, Symptome zu lindern und den Funktionsverlust zu antagonisieren. Dies geschieht durch Beeinflussung der Alveolitis und durch Abschmelzung der Granulome.

Angesichts der unbekannten Ätiologie der Sarkoidose handelt es sich dabei um keine kausale Therapie, sondern lediglich um eine Suppressionstherapie der verstärkten zellulären Immunreaktionen. Der natürliche Krankheitsverlauf, d.h. der Zeitpunkt, an dem die Krankheit nach Eliminierung des krankheitsauslösenden Faktors zur Ruhe kommt, kann durch eine Kortikosteroidtherapie nicht beeinflusst werden [19].

Beim Röntgentyp I ist in aller Regel eine Kortikosteroidtherapie entbehrlich. Gleiches gilt für das Löfgren-Syndrom (s. oben). Beim Röntgentyp II und III der Sarkoidose sollte man nur bei anhaltenden respiratorischen Symptomen und bei Lungenfunktionseinschränkungen behandeln. Eine Einschränkung besteht nur insofern, dass auch bei progredientem Röntgenbefund eine Therapienotwendigkeit bestehen kann, wenn dieser rasch und ausgeprägt erfolgt oder wenn Schrumpfungszeichen erkennbar werden.

Auch im Fibrosestadium wird eine Therapie regelhaft durchgeführt, da gelegentlich doch ein Ansprechen im Gefolge einer Langzeit-Therapie in niedriger Dosierung möglich ist.

Die Therapie der Wahl besteht aus der Gabe von oralem Prednison, initial ca. $0,5 \mathrm{mg} / \mathrm{kg}$ Körpergewicht, d.h. 20-40 mg täglich für 2-4 Wochen, dann stufenweise Reduktion in den nächsten 2 - 3 Monaten auf eine Erhaltungsdosis von 7,5 bis $15 \mathrm{mg}$ pro Tag. Diese Dosisreduktion muss individuell, das heißt abhängig vom initialen Ansprechen, der Schwere der Erkrankung und den Begleitumständen (Begleiterkrankungen wie Diabetes, Hypertonus) vorgenommen werden.

In aller Regel ist die Erhaltungsdosis nach 3 Monaten erreicht. Die alternierende Gabe der doppelten Dosis jeden 2. Tag ist grundsätzlich möglich. Die Therapiedauer beträgt 
Die Wirksamkeit einer oralen Kortikosteroidtherapie bei Sarkoidose II und III konnte durch eine Metaanalyse belegt werden.

Die Rezidivhäufigkeit kann bis zu 70\% betragen.

Cave: Routinemäßige BAL- oder CT-Kontrollen sollten unterbleiben.

Bei Herz- und Hirnbefall wird initial höher dosiert (1,0 mg/kg Körpergewicht). zwischen 6 und 12 Monaten. In Einzelfällen muss mehrere Jahre, gelegentlich sogar lebenslang behandelt werden.

Die Wirksamkeit einer oralen Kortikosteroidtherapie bei Sarkoidose II und III konnte durch eine Metaanalyse belegt werden; nach 6-24 Monaten fanden sich Verbesserungen des Röntgenthoraxbildes und der Lungenfunktion [19].

Die Therapie eines Rezidivs (die Häufigkeit beträgt 20 bis 70\%!) [20-22] wird entweder wiederum mit der üblichen Anfangsdosierung oder mit der Halbierung der Anfangsdosis und Verdoppelung der Therapiezeit (mindestens 1 Jahr) durchgeführt. Die gleichzeitige präventive Therapie mit INH bei Vorhandensein alter tuberkulöser Veränderungen wird nicht mehr generell empfohlen. Hier reichen klinische und radiologische Kontrollen aus, die wegen der Sarkoidose sowieso in Abständen von 3 bis 6 Monaten regelhaft durchgeführt werden müssen. Das Ansprechen auf die Therapie wird durch klinische Untersuchungen, Röntgen-Thorax und Lungenfunktion dokumentiert.

Die Indikation zur Durchführung einer Osteoporoseprophylaxe wird international strittig diskutiert. Die Gabe von Vitamin D und Kalzium bedarf wegen der Gefahr der Hyperkalzämie einer besonders strengen Indikationsstellung. Für die neueren Osteoporose-Präparate - wie Alendronsäure - sind bislang keine Studien verfügbar.

\subsection{Extrathorakale Manifestation}

Bei Befall von Herz, Niere, Hirn, Augen und Hyperkalzämie, sowie bei ausgeprägten Hautsarkoidosen, z. B. beim Lupus pernio (Abb. 5) besteht eine absolute Indikation zur Therapie. Seltenere wichtige Indikationen sind ausgeprägte Leber- und Milzsarkoidose mit Ikterus, sowie Knochensarkoidose mit funktionsstörender Fraktur. Bei Herz- und Hirnbefall wird initial höher dosiert (1,0 mg/kg Körpergewicht), in Gefahrsituationen i.v.-Therapie bis zu $300 \mathrm{mg}$ Cortison-Äquivalent pro Tag, meistens primär kombiniert mit Azathioprin.

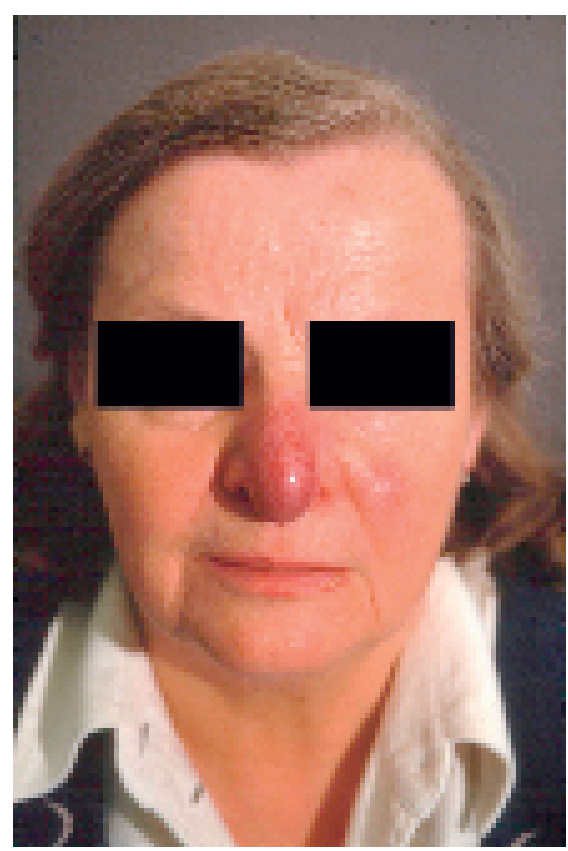

Abb. 5 Lupus pernio: Sarkoidosebefall der Nase. 
Bei Problemen mit einer CortisonMonotherapie ist die Kombination mit Azathioprin eine günstige, nebenwirkungsarme Alternative.
Die Dauertherapie muss vom klinischen Verlauf abhängig gemacht werden. Hier sind jeweils die Organmanifestationen mit ihren Störungen zu kontrollieren. Das betrifft sowohl die klinischen Untersuchungsbefunde, wie auch die Funktionen. Bei der Herzsarkoidose hat sich als Kontrollinstrument neben der Echokardiographie sowohl das MRT als auch das Langzeit-EKG als günstig erwiesen.

\subsection{Alternativen zur Kortikosteroidtherapie}

Alternative Medikamente kommen bei Sarkoidose nur bei schweren Kortikosteroidnebenwirkungen, bei Patienten, die nur auf hohe Dosen ansprechen oder solchen Patienten infrage, die eine Dauertherapie benötigen, bei der diese jedoch wegen einer vorbestehenden Osteoporose problematisch ist. Naturgemäß gibt es keine kontrollierten Studien zu diesen Therapien. Nach allgemeiner Ansicht wird meist zu einer niedrigdosierten Cortisontherapie Azathioprin hinzugefügt (100 bis $150 \mathrm{mg}$ pro Tag) [23]. Die Nebenwirkungen und Kontraindikationen sind bei derartiger Kombinationstherapie immer zu beachten. Eine gleich gute Wirkung wird in letzter Zeit auch der Kombination von niedrigdosiertem Cortison und Methotrexat (10-20 mg wöchentlich) zugebilligt [24,25]. Diese Kombination soll besonders gut bei Neurosarkoidose wirken. Gründe für diese besondere Organotropie sind nicht bekannt.

Cyclophosphamid und Chlorambucil sollten ausgesprochen therapierefraktären Fällen vorbehalten werden. Bei der Hautsarkoidose, aber auch bei anderen Organmanifestationen, hat sich Hydrochloroquin (200 bis 400 mg täglich) bewährt [26,27]. In den letzten Jahren wurde über erste erfolgreiche Therapieerfahrungen mit Infliximab berichtet [28]. Die Wirksamkeit von Pentoxifyllin [29] muss noch weiter untersucht werden.

Als unwirksam haben sich folgende Behandlungen bei Sarkoidose erwiesen: Cyclosporin A, Antituberkulotika, Antibiotika, Vitamine, Spironolacton, Immunstimulanzien.

\section{Schlussbemerkung}

Die Prävalenz von ca. 50-60/100000 zeigt, dass die Beschäftigung mit der Sarkoidose weiterhin lohnend ist. Differenzialdiagnostisch sind besonders atypische radiologische Lungensarkoidosen und die seltenen Organsarkoidosen eine permanente klinische Herausforderung. Es ist zu hoffen, dass es durch neue molekularbiologische Techniken in absehbarer Zukunft gelingt, den oder die Auslöser der Sarkoidose zu erkennen, um eine Kausaltherapie zu ermöglichen.

\section{Literatur}

1 Scharkoff T. Epidemiologie der Sarkoidose. Pneumologie 1993; 47: 588-592

2 Kirsten D. Sarkoidose in Deutschland. Pneumologie 1995; 48: 378 - 382

3 Hunninghake GW, Costabel U, Ando M et al. ATS/ERS/WASOG statement on sarcoidosis. Sarcoidosis Vasc Diffuse Lung Dis 1999; 16: 149-173

4 Drent M, Wirnsberger RM, Vries J de et al. Association of fatigue with an acute phase response in sarcoidosis. Eur Respir J 1999; 13: $718-722$

5 Deutsche Gesellschaft für Pneumologie. Empfehlungen zur Therapie der Sarkoidose. Pneumologie 1998; 52: 26-30

6 DeRemee RA. The roentgenolographic staging of sarcoidosis-historic and contemporary perspectices. Chest 1983; 83: $128-133$

7 Wurm K. Stadiengesetzlichkeit und Thoraxröntgenologie. In: Wurm K (Hrsg.). Sarkoidose. Stuttgart, New York: Thieme Verlag, 1983: 75-105

8 Winterbauer RH, Hutchinson JF. Use of pulmonary function tests in the management of sarcoidosis. Chest 1980; 78: 640-647

9 Costabel U. Sarcoidosis: clinical update. Eur Respir J 2001; 18 (Suppl. 32): 56s -68s

${ }^{10}$ Schulte W, Costabel U, Kirsten D. Kardiale Sarkoidose. Pneumologie 2003; 57: 752 - 760

${ }^{11}$ Chapman JT, Mehta AC. Bronchoscopy in sarcoidosis: diagnostic an therapeutic interventions. Curr Opin Pulm Med 2003; 9: 402-407

${ }^{12}$ Costabel U. Atlas der bronchoalveolären Lavage. Stuttgart: Thieme Verlag, 1994 
${ }^{13}$ Remy-Jardin M, Giraud F, Remy J et al. Pulmonary sarcoidosis: role of CT in the evaluation of disease activity and functional impairment and in prognosis assessment. Radiology 1994; 191: $675-680$

${ }^{14}$ Costabel U, Bois R du, Eklund A et al. Concensus Conference: Activity of Sarcoidosis. Eur Respir J 1994; 7: 624-627

${ }^{15}$ Sharma P, Smith I, Maguire G et al. Clinical value of ACE genotyping in diagnosis of sarcoidosis. Lancet 1997; 349: 1602-1603

${ }^{16}$ Müller-Quernheim J. Serum markers for the staging of disease activity of sarcoidosis and other interstitial lung diseases of unknown etiology. Sarcoidosis Vasc Diffuse Lung Dis 1998; 15: $22-37$

${ }^{17}$ Hillerdal G, Nou E, Ostermann K et al. Sarcoidosis: epidemiology and prognosis. A 15-year European study. Am Rev Respir Dis 1984; 130: 29-32

18 Viskum K, Vestbo J. Vital prognosis in intrathoracic sarcoidosis with special reference to pulmonary function and radiological stage. Eur Respir J 1993; 6: 349-353

19 Paramothayan S, Jones PW. Corticosteroid therapy in pulmonary sarcoidosis: a systematic review. JAMA 2002; 287: 1301 - 1307

${ }^{20}$ Gibson GJ, Prescott RJ, Muers MF et al. British Thoracic Sarcoidosis Study: effects on long-term corticosteroid treatment. Thorax 1996; 51: $238-247$

${ }^{21}$ Hunninghake GW, Gilbert S, Pueringer R et al. Outcome of the treatment for sarcoidosis. Am J Respir Crit Care Med 1994; 149: 893-898

${ }^{22}$ Gottlieb JE, Israel HL, Steiner RM et al. Outcome in sarcoidosis. The relationship of relapse to corticosteroid therapy. Chest 1997; 111: $623-631$

${ }^{23}$ Müller-Quernheim J, Kienast K, Held M et al. Treatment of chronic sarcoidosis with an azathioprine/prednisolone regimen. Eur Respr J 1999; 14: 1117 - 1122

${ }^{24}$ Baughman RP, Lower E. The effect of corticosteroid or methotrexate therapy on lung lymphocytes and macrophages in sarcoidosis. Am Rev Respir Dis 1990; 142: 1268-1271

${ }^{25}$ Baughman RP, Lower EE. A clinical approach to the use of methotrexate for sarcoidosis. Thorax 1999; 54: $742-746$

${ }^{26}$ Baltzan M, Mehta S, Kirham TH et al. Randomized trial of prolonged chloroquine therapy in advanced pulmonary sarcoidosis. Am J Respir Crit Care Med 1999; 160: 192 - 197

${ }^{27}$ Sharma OP. Effectiveness of chloroquine and hydroxychloroquine in treating selected patients with sarcoidosis with neurologic involvement. Arch Neurol 1998; 55: $1248-1254$

${ }^{28}$ Baughman RP, Lower EE. Infliximab for refractory sarcoidosis. Sarcoidosis Vasc Diffuse Lung Dis 2001; 18: 70-74

${ }^{29}$ Zabel P, Entzian P, Dahlhoff $\mathrm{K}$ et al. Pentoxifylline in the treatment of sarcoidosis. Am J Respir Crit Care Med 1997; 155: 1665-1669

Prof. Dr. D. Kirsten

Krankenhaus Großhansdorf · Zentrum für Pneumologie und Thoraxchirurgie

Wöhrendamm 80

22927 Großhansdorf

E-mail: d.kirsten@kh-grosshansdorf.de 

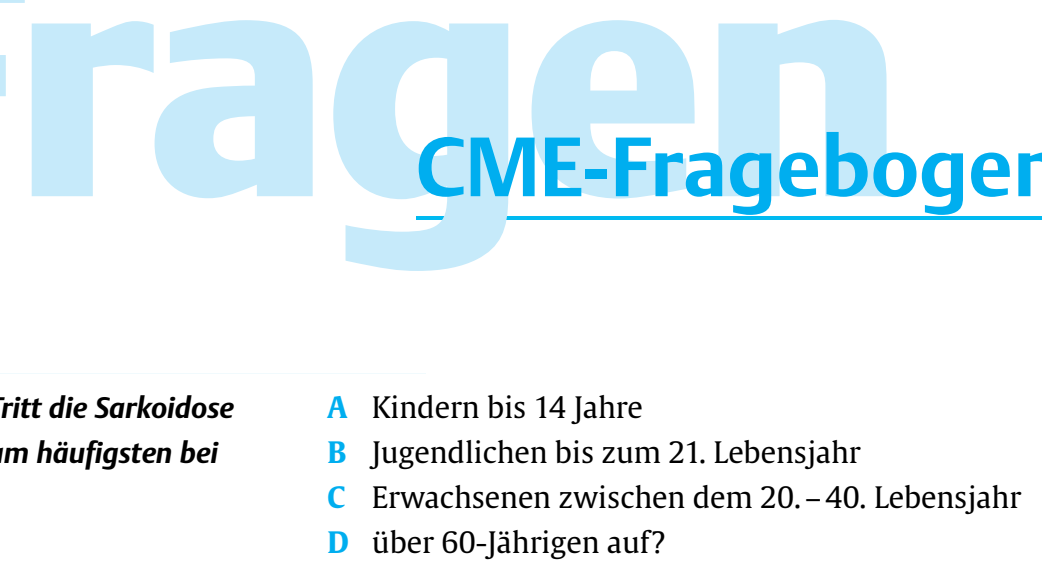

Tritt die Sarkoidose
A Kindern bis 14 Jahre
C Erwachsenen zwischen dem 20.-40. Lebensjahr
D über 60-Jährigen auf?

am häufigsten bei

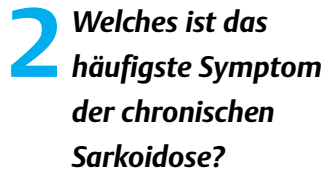

Bei welchem Rönt-

gentyp rechnet man am häufigsten mit spontaner Besserung?

Eine Kortisontherapie führt nicht zu
A Husten
B Atemnot
C Fieber
D Gewichtsabnahme
E Müdigkeit

mit bis zu $\quad$ E $70 \%$




\section{CME-Fragebogen}

\section{Welcher CT-Befund ist charakteristisch für die Sarkoidose?}
A Subpleurale Honigwaben
B „lymphangische“ Ausbreitung entlang der bronchovaskulären Bündel
C ausgedehntes Milchglasmuster
D einschmelzende Rundherde
E Pleuraverkalkungen
A Ciclosporin
B Methotrexat
C Infliximab
D Azathioprin
E Hydrochloroquin

Q Welches Medikament wird bei der Sarkoidosetherapie meist mit Kortison kombiniert?
A Ciclosporin
B Cyclophosphamid
C Infliximab
D Azathioprin
E Thalidomid

Welche diagnostische Methode erfreut sich im ambulanten Sektor der größten Beliebtheit?
A die BAL
B die Bronchialschleimhautbiopsie
C die Nasenschleimhautbiopsie
D das CT der Thoraxorgane
E die Rö-Thoraxaufnahme 


\section{Angaben zur Person}

\section{Evaluation}

Name, Vorname, akad. Titel:

Straße, Hausnummer: PLZ/Ort.

Ich bin Mitglied der Ärztekammer:

Jahr meiner Approbation:

Ich befinde mich in der Weiterbildung zum:

Ich habe eine abgeschlossene Weiterbildung in/für:

seit/Jahr der Facharztanerkennung:

Spezialisierung innerhalb des Fachgebiets: nein ja, welche?

Ich möchte folgende Zusatzbezeichnungen erwerben:

Ich habe folgende Zusatzbezeichnungen: seit:

Ich bin tätig als:

Assistenzarzt Oberarzt Chefarzt in folgender Klinik:

Niedergelassener Arzt, seit ___ im Ballungsraum im ländlichen Raum

Sonstiges (bitte eintragen):

Führen Sie in Ihrer Praxis diagnostische und therapeutische Auftragsleistungen im Bereich des Fortbildungsthemas durch?

nein ja, welche?

Bieten Sie in der Diagnostik und Therapie im Bereich des Fortbildungsthemas Besonderheiten in Ihrer Praxis/Klinik an?

nein ja, welche?

Machen Sie diese ggf. als Praxisbesonderheiten geltend?

nein ja, welche?

1. Wie viele Patienten diagnostizieren/behandeln Sie im Zusammenhang mit dem Fortbildungsthema pro Jahr?

2. Bitte benennen Sie die 3 wichtigsten für Sie noch offenen Probleme in der Diagnostik/Therapie des Fortbildungsthemas:

3. Bezüglich der Diagnostik/Therapie im Rahmen des Fortbildungsthemas fühle ich mich nach Studium des Beitrages in meiner Strategie bestätigt. hat sich meine Strategie folgendermaßen verändert - bitte benennen:

4. Wurden aus der Sicht Ihrer täglichen Praxis wichtige Aspekte des Themas
a) außer Acht gelassen?
nein
ja, welche?
b) zu knapp abgehandelt?
nein
ja, welche?
c) überbewertet?
nein
ja, welche? 


\begin{tabular}{l} 
Ihr Ergebnis \\
(wird vom Verlag ausgefüllt) \\
Sie haben___ von___ Fragen \\
richtig beantwortet und somit \\
$\square$ bestanden und 3 Punkte erworben. \\
$\square$ nicht bestanden. \\
Stuttgart, \\
\hline (Stempel/Unterschrift)
\end{tabular}

\section{Lernerfolgskontrolle}

Antworten zum Quiz

(nur eine Antwort pro Frage ankreuzen)
5. Etwa wieviel Prozent des Beitrages haben Ihnen

a) zur Auffrischung bereits bekannten Wissens gedient:

$$
<10 \%<25 \% \quad<50 \% \quad \geq 50 \%
$$

b) zur Erweiterung Ihres Spezialwissens gedient:

$$
<10 \%<25 \%<50 \% \quad \geq 50 \%
$$

6. Ergeben sich für Sie aus wirtschaftlichen Gründen Limitierungen im Einsatz von im Beitrag genannten Diagnose-/Therapieverfahren?

nein ja, welche?

7. Stehen Ihnen aus logistischen Gründen Limitierungen im Einsatz von im Beitrag genannten Diagnose-/Therapieverfahren nicht/nur eingeschränkt zur Verfügung? nein ja, welche?

8. Die Fragen lassen sich aus dem Studium des Beitrages allein beantworten.

\begin{tabular}{|c|c|c|c|c|c|c|c|c|}
\hline A & B & C & D & & A & B & C & D \\
\hline A & B & C & D & $\mathbf{E}$ & A & B & C & D \\
\hline A & B & C & D & $\mathbf{E}$ & A & B & C & D \\
\hline A & B & C & D & & A & B & C & D \\
\hline A & B & C & D & $\mathbf{E}$ & A & B & C & D \\
\hline
\end{tabular}
nur unter Zuhilfenahme zusätzlicher Literatur beantworten.

\section{Erklärung}

Ich versichere, dass ich die Beantwortung der Fragen selbst und ohne fremde Hilfe durchgeführt habe.

Ort/Datum

Unterschrift

Nichtabonnenten bitte hier CME-Wertmarke aufkleben bzw. Abonnentennummer eintragen
Bitte senden Sie den vollständig ausgefüllten Antwortbogen und einen an Sie selbst adressierten und ausreichend frankierten Rückumschlag an den Georg Thieme Verlag KG, CME, Joachim Ortleb, Postfach 301120, 70451 Stuttgart. Einsendeschluss ist der 30. Juni. 2006 (Datum des Poststempels). Die Zertifikate werden spätestens 14 Tage nach Erhalt des Antwortbogens versandt. Von telefonischen Anfragen bitten wir abzusehen.

\section{Teilnahmebedingungen der zertifizierten Fortbildung}

Für diese Fortbildungseinheit erhalten Sie 3 Fortbildungspunkte im Rahmen des Fortbildungszertifikates der Ärztekammern. Hierfür

- müssen $80 \%$ Fragen richtig beantwortet sein.

- muss die oben stehende Erklärung vollständig ausgefüllt sein. Unvollständig ausgefüllte Bögen können nicht berücksichtigt werden!

- muss Ihre Abonnentennummer im entsprechenden Feld des Antwortbogens angegeben oder eine CME-Wertmarke im dafür vorgesehenen Feld aufgeklebt sein.

\section{CME-Wertmarken für Nichtabonnenten}

CME-Wertmarken (für Teilnehmer, die die „Pneumologie“ nicht abonniert haben) können beim Verlag zu folgenden Bedingun- gen erworben werden: 6er-Pack Thieme-CME-Wertmarken, Preis $€$ 63,- inkl. MwSt., Artikel-Nr. 901916; 12er-Pack Thieme-CMEWertmarken, Preis $€ 99$,- inkl. MwSt., Artikel-Nr. 901917. Bitte richten Sie Bestellungen an: Georg Thieme Verlag KG, Kundenservice, Postfach 301120, 70451 Stuttgart.

\section{Wichtige Hinweise}

Die CME-Beiträge der „Pneumologie“ wurden durch die Ärztekammer Nordrhein anerkannt. Die „Pneumologie“ ist zur Vergabe der Fortbildungspunkte für diese Fortbildungseinheit berechtigt. Diese Fortbildungspunkte werden von anderen zertifizierenden Ärztekammern anerkannt. Die Vergabe der Fortbildungspunkte ist nicht an ein Abonnement gekoppelt! 